PROCEEDINGS OF THE

AMERICAN MATHEMATICAL SOCIETY

Volume 123, Number 1, January 1995

\title{
LARGE CARDINALS AND SMALL DOWKER SPACES
}

\author{
CHRIS GOOD
}

(Communicated by Franklin D. Tall)

\begin{abstract}
We prove that, if there is a model of set-theory which contains no first countable, locally compact, scattered Dowker spaces, then there is an inner model which contains a measurable cardinal.
\end{abstract}

A Hausdorff space is normal if, for every pair of disjoint closed sets $C$ and $D$, there is a pair of disjoint open sets, $U$ containing $C$ and $V$ containing $D$. A (normal) space is binormal if its product with the closed unit interval $I$ is normal. It is fair to say that the study of normality, in particular, the behaviour of normality in products and the difference between normality and binormality, has played a central role in point-set topology.

In 1951 Dowker [Do] introduced the notion of countable paracompactness and proved that a normal space is binormal iff it is countably paracompact. A space is (countably) paracompact if every (countable) open cover has a locally finite open refinement, however, the important point to note, as far as we are concerned, is that countable paracompactness is the difference between normality and binormality. A quick study of Dowker's paper demonstrates just how natural the definition is-indeed, countable paracompactness is not so much a generalization of paracompactness, as one in a list of related properties which act to preserve normality-type conditions in products with a compact, metrizable factor: $X \times I$ is respectively orthocompact, perfect, $\delta$-normal, normal, perfectly or hereditarily normal, or monotonically normal iff $X$ is (respectively) countably metacompact $[S]$, perfect (see [P, 4.9]), countably paracompact [M], normal and countably paracompact [Do], perfectly normal [Ka] and [P, 4.9], monotonically normal and semi-stratifiable [G, 5.22].

Normal spaces that are not countably paracompact have become known as Dowker spaces, and it is natural to ask whether such spaces exist.

In fact, Dowker spaces do exist, but the example [Ru2], together with its modifications, has unsatisfyingly large cardinality and cardinal functions. This has prompted the generic definition of small Dowker spaces, i.e., ones of small size or small cardinal functions. Small Dowker spaces also exist, but, as yet, only with the help of various set-theoretic assumptions. For example: when

Received by the editors November 2, 1992 and, in revised form, April 21, 1993; presented at the 7th Summer Conference in Honour of M. E. Rudin held in Madison, WI, June 1991.

1991 Mathematics Subject Classification. Primary 03E35, 03E55, 54D15, 54D20, 54G15.

Key words and phrases. Small Dowker space, normality, countable paracompactness, measurable cardinals, Covering Lemma. 
holds, there are first countable, locally compact ones-[dC], [Ru3]; if there is a Souslin tree, there are first countable, hereditarily separable ones-[Ru1]; under $C H$, there are first countable ones-[JKR]; under $M A+\left(\mathfrak{c}=\omega_{2}\right)+\diamond_{\omega_{2}}(E)$, there are locally compact, separable ones-[Ws], [Ru3]; under $\mathscr{P}(\mathfrak{c})$, which follows from $M A+\neg C H$ or the $P F A$, there are first countable ones-[Be]. Furthermore, Watson [Wt] has shown that the existence of a strongly compact cardinal implies the existence of a Dowker space that is of scattered length $\omega$.

There are also types of space which cannot be Dowker. Obviously metrizable, Lindelöf, (countably) paracompact, or (countably) metacompact spaces fail to be Dowker; perfect spaces are countably metacompact and so cannot be Dowker, which implies that Moore spaces are never Dowker; $G O$-spaces and monotonically normal spaces are not Dowker [Ru3]; normal, pseudocompact, or $D F C C$ spaces are countably compact [Tr] and so are not Dowker.

Is there a small Dowker space? Are there models of set-theory in which small Dowker spaces with certain properties cannot exist? In this paper we give a partial answer to the second of these questions. However, we feel that this result is misleading since we believe that the answer to the first question is probably yes. $^{1}$

The partial answer we give here is that large cardinals are needed for a model in which there are no first countable, locally compact Dowker spaces.

1. Theorem. If no inner model of set theory contains a measurable cardinal, then there is a first countable, locally countable, locally compact, zero-dimensional, collectionwise normal Dowker space which is scattered of length $\omega$ and is therefore both $\sigma$-discrete and weakly $\theta$-refinable.

2. Corollary. If every normal, Hausdorff space satisfying at least one of the above properties is countably paracompact, then some inner model contains a measurable cardinal.

(It should be pointed out that the collectionwise normal case is only interesting when some other property from the list is added, since Rudin's Dowker space [Ru2] is collectionwise normal.)

Of course, the motivation for Theorem 1 is Fleissner's result [F1] that large cardinals are needed for a model in which there are no normal, non-metrizable Moore spaces, and the steps in the proof are much the same: we start from the assumption that no inner model of set-theory contains a measurable cardinal, and derive enough combinatorics to allow us to construct a typical (see [Ru3]) first countable, locally compact Dowker space.

Our notation and terminology are standard, taken from [E], [Ku], and [KV]. However, for a function $f: A \rightarrow B$, we denote $\{f(x): x \in C \subseteq A\}$ by $f[C]$.

3. Definition. For an infinite cardinal $\kappa$ and a subset $A \subseteq \kappa^{+}, \square_{\kappa}(A)$ is the assertion that there is a sequence $\left\{C_{\alpha}: \alpha \in \kappa^{+}\right.$and $\left.\operatorname{LIM}(\alpha)\right\}$ such that:

(i) $C_{\alpha}$ is club in $\alpha$;

(ii) if $\operatorname{cf}(\alpha)<\kappa$, then $\operatorname{otp}\left(C_{\alpha}\right)<\kappa$;

(iii) if $\beta<\alpha$ is a limit point of $C_{\alpha}$, then $\beta \notin A$ and $C_{\beta}=\beta \cap C_{\alpha}$.

\footnotetext{
${ }^{1}$ Added in proof: Indeed Balogh has recently proved the existence of a scattered Dowker space with the cardinality of the continuum.
} 
We write $\square_{\kappa}$ for $\square_{\kappa}(\varnothing)$. It it clear from the definition that $\square_{\kappa}$ is really a statement about $\kappa^{+}$. For more about $\square_{\kappa}$ see [De].

4. Definition. A stationary subset $E$ of some uncountable cardinal $\lambda$ is said to be non-reflecting if, for every $\alpha<\lambda, \alpha \cap E$ is non-stationary in $\alpha$.

Notice that, if $\square_{\kappa}(E)$ and if $E$ is stationary in $\kappa^{+}$, then $E$ must be nonreflecting. (To see this, suppose to the contrary that $\alpha \cap E$ is stationary in $\alpha$ for some $\alpha$ in $\kappa^{+}$; as $C_{\alpha}$ is club in $\alpha$ there is some limit point $\beta$ in $\alpha \cap E \cap C_{\alpha}$, so $\beta$ is in $E$, which contradicts statement (iii) of Definition 3.) Every stationary subset of $\omega_{1}$ is non-reflecting, but the existence of non-reflecting stationary subsets of ordinals greater than $\omega_{1}$ is not guaranteed. (In fact, it is clear from what follows that large cardinals are needed for a model in which there are no non-reflecting stationary sets.)

We shall also need:

5. Definition. A cardinal $\kappa$ is said to be a strong limit cardinal if $(\forall \lambda<\kappa)$ $\left(2^{\lambda}<\kappa\right)$.

Now, $K$ is the Core model defined by Dodd and Jensen [DJ]. $K$ contains the constructible universe $L$ and is contained in $V$. Here we need only the fact that, combinatorially, $K$ is very similar to $L$; in particular, $\square_{\kappa}$ holds in $K$ for all infinite cardinals $\kappa$ [Dd].

6. The Covering Lemma for $V$ and $K . \operatorname{Cov}(V, K)$ is the assertion that, if $X$ is any uncountable set of ordinals (in $V$ ), then there is a set $Y$ in $K$ such that $X$ is a subset of $Y$ and $|Y|=|X|$ (in $V)$.

$\operatorname{Cov}(V, K)$ implies that $V$ is very similar to $K$. This need not always be the case, for example when $0^{\#}$ exists (see [De]), however, we have the following theorem due to Dodd and Jensen [DJ].

7. Theorem (Dodd and Jensen). If no inner model of set theory contains a measurable cardinal, then the Covering Lemma for $V$ and $K$ holds.

The proof of this result is extremely long and requires a detailed knowledge of the fine structure of $K$, however, it is the starting point for Theorem 1 . We shall now proceed from the Covering Lemma, through a series of lemmas, to the construction of Example 17, which will complete the proof. The crucial step is to notice that $\diamond$ on a non-reflecting stationary subset of $\kappa^{+}$is enough to give normality.

The proof of Lemma 8 is essentially contained in the proof of Theorem 8.3 of [F2].

8. Lemma (Various). $\operatorname{Cov}(V, K)$ implies that there exists an uncountable strong limit cardinal $\kappa$ of countable cofinality, such that $2^{\kappa}=\kappa^{+}$and $\square_{\kappa}$.

9. Definition. For a stationary subset $S$ of $\kappa^{+}, \nabla_{\kappa^{+}}(S)$ is the assertion that there exists a sequence $\left\{S_{\alpha}: \alpha \in S\right\}$, such that $S_{\alpha}$ is a subset of $\alpha$, and, whenever $X$ is a subset of $\kappa^{+}$, the set

$$
\left\{\alpha \in S: X \cap \alpha=S_{\alpha}\right\}
$$

is stationary in $\kappa^{+}$. 
Unlike $\square_{\lambda}, \diamond_{\lambda}$ is a statement about $\lambda$; we write $\nabla_{\kappa^{+}}$here to emphasise the connection between $\square_{\kappa}$ and $\nabla_{\kappa^{+}}$. Notice that for any subset $A$ of $\kappa^{+}$, $\diamond_{\kappa^{+}}(A)$ implies that $A$ has to be stationary in $\kappa^{+}$.

10. Definition. For any (regular) cardinal $\lambda$ let

$$
W(\lambda)=\{\alpha \in \lambda: \operatorname{cf}(\alpha)=\omega\}
$$

In particular, let $W$ denote $W\left(\kappa^{+}\right)$. It is easy to see that $W$ (or indeed $W(\lambda)$ ) is stationary.

The next lemma is a slight rewording of Exercise IV.8 in [De], its proof is a modification of [De, Lemma IV.2.8]. Lemma 12 is exactly [De, IV.2.10].

11. Lemma. Suppose that the conclusion of Lemma 8 holds, then $\nabla_{\kappa^{+}}(W)$.

12. Lemma. If both $\square_{\kappa}$ and $\diamond_{\kappa^{+}}(W)$, then there exists a stationary subset $E$ of $W$ such that both $\square_{\kappa}(E)$ and $\diamond_{\kappa^{+}}(E)$.

Because both $\square_{\kappa}(E)$ and $\vartheta_{\kappa^{+}}(E)$ hold, we do indeed have $\diamond$ on a nonreflecting stationary subset of $\kappa^{+}$. In the next two lemmas we deduce the a-type principle which is used in the construction of the example. The proof of Lemma 14 is a standard coding argument (see, for example, [Ws]).

13. Definition. $\diamond_{\kappa^{+}}(E, 2)$ is the assertion that there exists a sequence $\left\{\left\langle S_{\alpha}, T_{\alpha}\right\rangle: \alpha \in E\right\}$, such that both $S_{\alpha}$ and $T_{\alpha}$ are subsets of $\alpha$, and, whenever $X$ and $Y$ are subsets of $\kappa^{+}$, the set

$$
\left\{\alpha \in S: X \cap \alpha=S_{\alpha}, Y \cap \alpha=T_{\alpha}\right\}
$$

is stationary in $\kappa^{+}$.

14. Lemma. $\diamond_{\kappa^{+}}(E, 2)$ is equivalent to $\diamond_{\kappa^{+}}(E)$.

The proof of Lemma 14 mimics the folklore proof that $\diamond$ is equivalent to $\diamond\left(\omega_{1}, 2\right)$, a similar proposition is to be found in [Ws].

The axiom we actually use to construct the example is something which we call $\boldsymbol{\$}_{\kappa^{+}}(E, 2)$, after Ostaszewski's \& [O]. The proof that $\diamond_{\kappa^{+}}(E, 2)$ implies $\boldsymbol{\$}_{\kappa^{+}}(E, 2)$ is essentially the same as the proof that $\diamond$ implies $\$$ and is therefore omitted.

15. Definition. $\boldsymbol{\beta}_{\kappa^{+}}(E, 2)$ is the assertion that for each limit ordinal $\alpha$ in $E$ of countable cofinality there exists a pair $\left\langle S_{\alpha}, T_{\alpha}\right\rangle$ of $\omega$-sequences, each cofinal in $\alpha$, such that, whenever $X$ and $Y$ are subsets of $\kappa^{+}$of size $\kappa^{+}$, there is some $\alpha \in \kappa^{+}$with $S_{\alpha} \subseteq X$ and $T_{\alpha} \subseteq Y$. (In fact, the set of such $\alpha$ forms a stationary set.)

16. Lemma. $\nabla_{\kappa^{+}}(E, 2)$ implies $\boldsymbol{\$}_{\kappa^{+}}(E, 2)$.

17. Example. $\left(\boldsymbol{\$}_{\kappa^{+}}(E, 2)\right)$ There is a first countable, locally countable, locally compact, strongly zero-dimensional, collectionwise normal Dowker space, which is scattered of length $\omega$ and is therefore both $\sigma$-discrete and weakly $\theta$-refinable. Proof. For each limit ordinal $\alpha$ in $E$, let $\left\langle S_{\alpha}, T_{\alpha}\right\rangle$ be as given by $\boldsymbol{q}_{\kappa^{+}}(E, 2)$. Without loss of generality, we may assume that $S_{\alpha} \cap T_{\alpha}$ is empty. Partition $S_{\alpha}$ into disjoint $\omega$-sequences $S_{\alpha, n}, n \in \omega$, and $T_{\alpha}$ into disjoint $\omega$-sequences, $T_{\alpha, n}$. Let

$$
R_{\alpha, n}=S_{\alpha, n} \cup T_{\alpha, n} \quad \text { and } \quad R_{\alpha}=\bigcup_{n \in \omega} R_{\alpha, n}=S_{\alpha} \cup T_{\alpha},
$$


index $R_{\alpha, n}$ increasingly as $\left\{\beta_{\alpha, n, j}: j \in \omega\right\}$ and index $R_{\alpha}$ increasingly as $\left\{\beta_{\alpha, k}: k \in \omega\right\}$.

The point set for the space $X$ is $\kappa^{+} \times \omega$, and the map $\pi$ is the natural projection, $\pi((\alpha, n))=\alpha$, from $X$ onto $\kappa^{+}$with its usual order topology.

Inductively we define a neighbourhood base $\mathscr{B}_{x}$ of compact, clopen subsets at each point $x$. The topology $\mathscr{T}$ on $X$ is generated by $\bigcup_{x \in X} \mathscr{B}_{x}$.

Let $x=(\alpha, n)$. If either $n=0, \alpha=\beta+1$ for some $\beta$, or $\alpha$ is not in $E$, then $x$ is isolated, i.e., $\mathscr{B}_{x}=\{\{x\}\}$. For the induction, let $x=(\alpha, n+1)$, and suppose that $\mathscr{B}_{(\beta, m)}$ has been defined for each $(\beta, m)$, where $\beta<\alpha$ and $m<n+1$, and suppose that $\alpha$ is a limit ordinal in $E$. Recall that $R_{\alpha, n}$ is indexed increasingly as $\left\{\beta_{\alpha, n, j}: j \in \omega\right\}$. For each point $y(j)=\left(\beta_{\alpha, n, j}, n\right)$, $j \geq 1, \beta_{\alpha, n, j}$ occurs as some $\beta_{\alpha, k(n, j)}$ in the enumeration of $R_{\alpha}$. Choose $B_{y(j)}$ in $\mathscr{B}_{y(j)}$ such that $\pi\left[B_{y(j)}\right]$ is a subset of $\left(\beta_{\alpha, k(n, j)-1}, \beta_{\alpha, k(n, j)}\right]$, and define $\mathscr{B}_{x}$ to be the collection of sets $\{B(x, k)\}_{k \in \omega}$ such that

$$
B(x, k)=\{x\} \cup \bigcup_{j \geq k} B_{y(j)} \quad \text { for } k \in \omega .
$$

Let $\mathscr{T}$ be the topology generated by the $\mathscr{B}_{x}$.

With this topology, points of $X$ are either isolated or have a neighbourhood homeomorphic to the ordinal space $\omega^{m}+1$, for some $m \leq n$. Therefore, $(X, \mathscr{T})$ is $T_{3}$, first countable, locally countable, locally compact, zerodimensional, and locally metrizable. It is also scattered of height $\omega$, and hence $\sigma$-discrete, and, by a result of [N1], weakly $\theta$-refinable.

Claim 1. The subspace $X_{\alpha}=\alpha \times \omega$ is metrizable for all $\alpha \in \kappa^{+}$.

Proof of Claim 1. The proof is by induction. Assume that $X_{\beta}$ is metrizable for all $\beta \in \alpha$.

Since $E$ is a non-reflecting stationary set, if $\alpha$ is a limit ordinal (either in $E$ or not), or $\alpha \leq \omega_{1}$, then there is a sequence $\left\{\alpha_{\gamma}: \gamma \in \theta \leq \alpha\right\}$, which is both closed and cofinal in $\alpha-E$. But then $X(\gamma)=\left(\alpha_{\gamma}, \alpha_{\gamma+1}\right] \times \omega$ is a metrizable subspace of $X$ and the collection $\{X(\gamma): \gamma \in \theta\}$ is a partition of $X_{\alpha}$ into disjoint, clopen, metrizable sets. Hence $X_{\alpha}$ is metrizable.

Now suppose that $\alpha=\beta+1$. Without loss of generality, we may assume that $\beta$ is a limit ordinal. If $\beta$ is not in $E$, then the two sets $X_{\beta}$ and $\{(\beta, n)$ : $n \in \omega\}$ partition $X_{\alpha}$ into disjoint, clopen, metrizable sets, and we are done. So, assume that $\beta$ is an element of $E$.

By construction, $\left\{B_{j}\right\}_{j \in \omega}$, where $B_{j}=B((\beta, j), 1)$, forms a disjoint collection of clopen, metrizable subsets of $X_{\alpha}$. Furthermore, if $x_{j}$ is any point of $B_{j}$, then the set $\left\{\pi\left(x_{j}\right)\right\}_{j \in \omega}$ forms an $\omega$-sequence, cofinal in $\beta$ (though not necessarily indexed in increasing order), so the only possible limit point of the sequence $\left\{x_{j}\right\}_{j \in \omega}$ is $(\beta, k)$ for some $k$ in $\omega$. This is impossible since $\left\{B_{j}\right\}_{j \in \omega}$ is a disjoint collection and is, therefore, a discrete collection. But now the two sets

$$
B=\bigcup_{j \in \omega} B_{j} \quad \text { and } Y=X_{\alpha}-B
$$

partition $X_{\alpha}$ into disjoint, clopen, metrizable subspaces, and again $X_{\alpha}$ is metrizable.

That $X$ is a Dowker space now follows from the familiar 
Claim 2. Every subset $A$ of $X$ of size $\kappa^{+}$has a limit point, and there are no two disjoint closed subsets, both of size $\kappa^{+}$.

Proof of Claim 2. $|A|=\kappa^{+}$, so for some $n \in \omega$,

$$
A_{n}=A \cap\left(\kappa^{+} \times\{n\}\right)
$$

has size $\kappa^{+}$. By $\boldsymbol{\beta}_{\kappa^{+}}(E, 2), R_{\alpha} \times\{n\}$, and hence $R_{\alpha, n} \times\{n\}$, is contained in $A_{n}$, for some $\alpha$ in $E$, so $A$ has a limit point in $\kappa^{+} \times\{n+1\}$. In fact, since $\kappa^{+}$is a regular cardinal, $A$ has $\kappa^{+}$limit points in $\kappa^{+} \times\{n+1\}$.

Now let $C$ and $D$ be closed subsets of $X$ of cardinality $\kappa^{+}$. From the previous paragraph it is clear that

$$
\left|C \cap\left(\kappa^{+} \times\{n\}\right)\right|=\left|D \cap\left(\kappa^{+} \times\{n\}\right)\right|=\kappa^{+},
$$

for some $n$. By $\boldsymbol{\beta}_{\kappa^{+}}(E, 2)$, there is an $\alpha$ in $E$ for which both $S_{\alpha} \times\{n\}$ is a subset of $C \cap\left(\kappa^{+} \times\{n\}\right)$ and $T_{\alpha} \times\{n\}$ is a subset of $D \cap\left(\kappa^{+} \times\{n\}\right)$. Therefore, $S_{\alpha, n} \times\{n\}$ is a subset of $C$ and $T_{\alpha, n} \times\{n\}$ is a subset of $D$. Since $C$ and $D$ are both closed, $(\alpha, n+1)$ is in both $C$ and $D$. Hence $C$ and $D$ are not disjoint-proving the claim.

Normality is immediate from Claims 1 and 2: Let $C$ and $D$ be disjoint closed sets. By Claim 2, at least one, $C$ say, has size less than $\kappa^{+}$, and there is some $\alpha=\beta+1$ in $\kappa^{+}$such that $X_{\alpha}=\alpha \times \omega$ contains $C$. By Claim 1 and the fact that $\alpha$ is a successor ordinal, $X_{\alpha}$ is a clopen metrizable subspace of $X$ in which $C$ and $D \cap X_{\alpha}$ can be separated by disjoint open sets. The normality of $X$ follows easily. In fact, if $\mathscr{D}$ is a discrete collection of closed subsets of $X$, then, since every subset of size $\kappa^{+}$has a limit point, $\mathscr{D}$ has size less than $\kappa^{+}$, and at most one member of $\mathscr{D}$ has size $\kappa^{+}$. Therefore, since $X_{\alpha}$ is metrizable for all $\alpha<\kappa^{+}, X$ is collectionwise normal.

A Tychonoff space $Y$ is strongly zero-dimensional iff for every pair of completely separated subset, $A$ and $B$, of $Y$ there is a clopen set $K$ which misses $B$ but contains $A$. The strong zero-dimensionality of $X$ also follows from Claims 1 and 2: Suppose that $A$ and $B$ are subsets of $X$ which are completely separated by the function $f: X \rightarrow[0,1]$ and that $f[A]=\{0\}$ and $f[B]=\{1\}$. The sets $f^{-1}[[0,1 / 4]]$ and $f^{-1}[[3 / 4,1]]$ are disjoint closed sets containing $A$ and $B$, respectively, so, arguing as above, there is a clopen subeset $X_{\alpha}$ of $X$ which misses $B$ say. Now $X_{\alpha}$ is a metrizable, locally compact, zero-dimensional subspace of $X$ and is therefore strongly zero-dimensional (see [E, 6.2.10]). Strong zero-dimensionality now follows easily.

To see that $X$ is a Dowker space, we show that it is not countably metacompact. By Dowker's characterization of countable metacompactness [Do], it is enough to show that there is a decreasing sequence $\left\{D_{n}\right\}_{n \in \omega}$ of closed sets with empty intersection such that whenever $\left\{U_{n}\right\}_{n \in \omega}$ is a decreasing sequence of open sets, each $U_{n}$ containing $D_{n}$, the intersection of the $U_{n}$ is non-empty.

Let $D_{n}=\kappa^{+} \times[n, \omega) .\left\{D_{n}\right\}_{n \in \omega}$ is a decreasing sequence of closed subsets of $X$ with empty intersection. If $U_{n}$ is any open set containing $D_{n}$, then $D_{n}$ and $X-U_{n}$ are disjoint closed subsets. $D_{n}$ has cardinality $\kappa^{+}$, so, by Claim 2 ,

$$
\left|X-U_{n}\right| \leq \kappa \text { and }\left|\bigcup_{n \in \omega}\left(X-U_{n}\right)\right|=\left|X-\bigcap_{n \in \omega} U_{n}\right|=\kappa .
$$

But this means that $\bigcap_{n \in \omega} U_{n}$ is non-empty, so $X$ is not countably metacompact. 
This completes the proof of Theorem 1. The same construction works if we assume either $V=L$ or $V=K$ (in which case we may take $\kappa=\omega$ ) or the non-existence of $0^{\#}$ (instead of 'no inner model contains a measurable cardinal').

18. Lemma. The space $X$ is not hereditarily normal.

Proof. Consider the subspace $Y=\kappa^{+} \times 2$, i.e., the first two levels of $X$. Divide the top level $\kappa^{+} \times\{1\}$ of $Y$ into two disjoint subsets, $C$ and $D$, each of cardinality $\kappa^{+}$, so that $C$ and $D$ are disjoint closed subsets of $Y$. Let $U$ and $V$ be any two closed neighbourhoods of $C$ and $D$, respectively; then both

$$
\left|U \cap\left(\kappa^{+} \times\{0\}\right)\right|=\kappa^{+} \text {and }\left|V \cap\left(\kappa^{+} \times\{0\}\right)\right|=\kappa^{+} .
$$

By $\boldsymbol{\beta}_{\kappa^{+}}(E, 2), U$ and $V$ have a common limit point in $\kappa^{+} \times\{1\}$, which must be in either $C$, which is a subset of $U$, or $D$, which is a subset of $V$. Therefore, $U$ and $V$ have a common element in $\kappa^{+} \times\{0\}$. Hence $Y$ is not normal.

Since $E$ is non-reflecting, this subspace $Y$ is $<\kappa^{+}$-collectionwise Hausdorff (i.e., discrete collections of points of size less than $\kappa^{+}$can be separated by pairwise disjoint open sets) but not collectionwise Hausdorff. Tall has asked [vMR, ?47] whether it is consistent (assuming large cardinals) for every first countable space which is $\omega_{1}$-collectionwise Hausdorff to be collectionwise Hausdorffpresumably he has the above example in mind.

While pseudocompact spaces are never Dowker, the following lemma says that every continuous, $\mathbb{R}$-valued function on $X$ is eventually constant.

19. Lemma. If $f: X \rightarrow \mathbb{R}$ is any continuous function, then there is a $\gamma \in \kappa^{+}$ such that $f$ is constant on $X-X_{\gamma}$.

Proof. For each $n \in \omega$, let $A_{n}$ be the set $f^{-1}[[n, n+1]]$. Pick some $n$ for which $A_{n} \cap\left(\kappa^{+} \times\{0\}\right)$ has size $\kappa^{+}$. Inductively define subsets $B_{k}$ of $A_{n}$ such that: $B_{0}$ is $A_{n}$; if $f\left[B_{k}\right]$ is the interval $\left[b, b+1 / 2^{k}\right]$, then $f\left[B_{k+1}\right]$ is either the interval $\left[b, b+1 / 2^{k+1}\right]$ or the interval $\left[b+1 / 2^{k+1}, b+1 / 2^{k}\right]$; and $B_{k} \cap\left(\kappa^{+} \times\{0\}\right)$ has size $\kappa^{+}$. Since $f\left[A_{n}\right]$ is compact, $\cap f\left[B_{k}\right]$ is non-emptyin fact, it contains just one real, $r$ say.

For each $n \in \omega$ let $R_{n}$ be the closed set $f^{-1}[[r-1 / n, r+1 / n]]$. Now each $R_{n}$ has cardinality $\kappa^{+}$, and it follows from Claim 2 of 17 that $r$ is the only point in $\mathbb{R}$ with this property. This implies not only that $r$ is in the $f$-image of $\kappa^{+} \times\{0\}$, but that there is some $\gamma_{0} \in \kappa^{+}$for which $f \uparrow\left(\gamma_{0}, \kappa^{+}\right) \times\{0\}$ is the constant function taking the value $r$. By the continuity of $f$, for each $n$ there is a $\gamma_{n}$ such that $f \uparrow\left(\gamma_{n}, \kappa^{+}\right) \times\{n\}$ is the constant function taking the value $r$.

Let $\gamma$ be $\sup \gamma_{n}$.

A space is realcompact if it is homeomorphic to a closed subspace of a Tychonoff product $(0,1)^{\lambda}$ of the open unit interval. Since pseudocompact, realcompact are compact, and since pseudocompact, normal spaces are countably paracompact, it is reasonable to ask about the existence of realcompact Dowker spaces. However, from Lemma 19 it follows that

\section{Lemma. $X$ is not realcompact.}

Proof. Let $Z_{\gamma}$ be the subset $\left(\gamma, \kappa^{+}\right) \times \omega$ of $X$, and let $\mathscr{Z}$ be the collection of all such subsets. $\mathscr{Z}$ is a collection of functionally closed subsets of $X$ and 
the ultrafilter $\mathscr{U}$ of functionally closed sets generated by $\mathscr{Z}$ has the countable intersection property. However, $\cap \mathscr{U}$ is empty and, by [E, 3.11.11], $X$ is not realcompact.

The normal Moore space conjecture (that all normal Moore spaces are metrizable) has been solved modulo the existence of large cardinals: In [N2] Nyikos shows that all normal Moore spaces are metrizable assuming the $P M E A$, the consistency of which is implied by the consistency of a strongly compact cardinal. Fleissner's result, mentioned above, shows that some large cardinal assumption is necessary for this result. Let us conclude with a number of observations which indicate why a result for small Dowker spaces analogous to Nyikos's seems unlikely.

We start with a theorem from [NP].

21. Theorem (Nyikos and Purisch). Let $X$ be a locally countable, regular space of countable scattered length. If $X$ is hereditarily collectionwise Hausdorff, then $X$ is paracompact.

It is immediate that

22. Corollary. There are no locally countable, hereditarily collectionwise Hausdorff Dowker spaces of countable scattered length.

From Nyikos's provisional solution to the normal Moore space problem [N2] and from [Ta, 2.20] we obtain

23. Corollary. Let $X$ be a locally countable, hereditarily normal space of countable scattered length.

$(P M E A)$ If $X$ has character less than the continuum, then $X$ is not a Dowker space.

$(V=L)$ If $X$ is first countable or is locally compact, then $X$ is not a Dowker space.

24. Fact. PMEA is consistent with the existence of first countable Dowker spaces: Adding one Cohen real gives a model in which there is Souslin tree (see [Ro, p. 311] and hence a first countable Dowker space [Ru1]. If $\kappa$ is strongly compact, then adding $\kappa$ many Random reals to this model preserves the Souslin tree [KT] (and hence the Dowker space), but also implies the PMEA.

Since $P F A$ implies $M A+\left(\mathfrak{c}=\omega_{2}\right)$, [Be] implies

25. Theorem. $(P F A)$ There is a first countable Dowker space.

On the other hand we have, essentially from [Bl]:

26. Theorem. $(P F A)$ No locally compact, $\sigma$-discrete space of cardinality smaller than $\mathfrak{c}=\omega_{2}$ can be a Dowker space.

Finally let us recall from above that Watson [Wt] has constructed a Dowker space directly from the assumption that there exist a strongly compact cardinal.

\section{ACKNOWLEDGMENTS}

The author would like to thank Frank Tall for suggesting that there might be a connection between large cardinals and Dowker spaces. This research was funded by a SERC studentship. 


\section{REFERENCES}

[Bl] Z. Balogh, Locally nice spaces under Martin's axiom, Comment. Math. Univ. Carolin. 24 (1983), 63-87.

[Be] M. G. Bell, On the combinatorial principal $P(\mathfrak{c})$, Fund. Math. 114 (1981), 149-157.

[dC] P. de Caux, A collectionwise normal weakly $\theta$-refinable Dowker space which is neither irreducible nor realcompact, Topology Proc. 1 (1976), 67-77.

[De] K. Devlin, Constructability, Springer-Verlag, Berlin, 1984.

[Dd] A. J. Dodd, Core models, J. Symbolic Logic 48 (1983), 78-90.

[DJ] A. J. Dodd and R. Jensen, The covering lemma for K, Ann. Math. Logic 22 (1982), 1-30.

[Do] C. H. Dowker, On countably paracompact spaces, Canad. J. Math. 3 (1951), 219-224.

[E] R. Engelking, General topology, Heldermann Verlag, Berlin, 1989.

[F1] W. G. Fleissner, If all normal Moore spaces are metrizable then there is an inner model with a measurable cardinal, Trans. Amer. Math. Soc. 273 (1982), 365-373.

[F2] - The normal Moore space conjecture and large cardinals, Handbook of Set-Theoretic Topology (K. Kunen and J. E. Vaughan, eds.), North-Holland, Amsterdam, 1984.

[G] G. Gruenhage, Generalized metric spaces, Handbook of Set-Theoretic Topology (K. Kunen and J. E. Vaughan, eds.), North-Holland, Amsterdam, 1984.

[JKR] I. Juhász, K. Kunen, and M. E. Rudin, Two more hereditarily separable non-Lindelöf spaces, Canad. J. Math. 28 (1976), 998-1005.

[Ka] M. Katětov, Complete normality of Cartesian products, Fund. Math. 38 (1948), 271-274.

[Ku] K. Kunen, Set theory, an introduction to independence proofs, North-Holland, Amsterdam, 1984.

[KT] K. Kunen and F. D. Tall, Between Martin's axiom and Souslin's hypothesis, Fund. Math. 102 (1979), 173-181.

[KV] K. Kunen and J. E. Vaughan, eds., Handbook of set-theoretic topology, North-Holland, Amsterdam, 1984.

[M] J. Mack, Countable paracompactness and weak normality properties, Trans. Amer. Math. Soc. 148 (1970), 265-272.

[vMR] J. van Mill and G. M. Reed, eds., Open problems in topology, North-Holland, Amsterdam, 1990.

[N1] P. J. Nyikos, Covering properties on $\sigma$-scattered spaces, Topology Proc. 2 (1977), 509-541.

[N2] A provisional solution to the normal Moore space problem, Proc. Amer. Math. Soc. 78 (1980), 429-435.

[NP] P. J. Nyikos and S. Purisch, Monotone normality and paracompactness in scattered spaces, Papers on General Topology and Related Category Theory and Topological Algebra, Ann. New York Acad. Sci., vol. 552, New York Acad. Sci., New York, 1989, pp. 124-137.

[O] A. J. Ostaszewski, On countably compact, perfectly normal spaces, J. London Math. Soc. 14 (1976), 505-516.

[P] T. C. Przymusiński, Products of normal spaces, Handbook of Set-Theoretic Topology (K. Kunen and J. E. Vaughan, eds.), North-Holland, Amsterdam, 1984.

[Ro] J. Roitman, Basic $S$ and $L$, Handbook of Set-Theoretic Topology (K. Kunen and J. E. Vaughan, eds.), North-Holland, Amsterdam, 1984.

[Ru1] M. E. Rudin, Countable paracompactness and Souslin's problem, Canad. J. Math. 7 (1955), 543-547.

[Ru2] _ A normal space for which $X \times I$ is not normal, Fund. Math. 72 (1971), 179-186.

[Ru3] _ Dowker spaces, Handbook of Set-Theoretic Topology (K. Kunen and J. E. Vaughan, eds.), North-Holland, Amsterdam, 1984.

[S] B. M. Scott, Toward a product theory for orthocompactness, Studies in Topology, Academic Press, New York, 1975, pp. 517-537.

[Ta] F. D. Tall, Normality versus collectionwise normality, Handbook of Set-Theoretic Topology (K. Kunen and J. E. Vaughan, eds.), North-Holland, Amsterdam, 1984. 
[Tr] I. J. Tree, Pseudocompactness and chain conditions, Ph.D., University of Oxford, London, 1991.

[Wt] W. S. Watson, A construction of a Dowker space, Proc. Amer. Math. Soc. 109 (1990), 835-841.

[Ws] W. Weiss, Small Dowker spaces, Pacific J. Math. 91 (1981), 485-492.

WADHAM COLlege, OXFord UNIVERSITY, OXFord, OX1 3PN, UNITED KINGDOM

E-mail address: cgoodemaths.ox.ac.uk 